\title{
„Existiert überhaupt eine Methode, die das bewirkt, was man erreichen möchte?"
}

\author{
Alexandra Carpentier im Gespräch mit Timo de Wolff
}

\begin{abstract}
Die Mathematikerin Alexandra Carpentier ist im September 2020 mit dem von Kaven-Ehrenpreis 2020 der Deutschen Forschungsgemeinschaft (DFG) für Nachwuchsforscher ausgezeichnet worden. Den Preis erhielt sie als Leiterin einer Forschergruppe im Emmy Noether-Programm der DFG. Seit 2017 ist die 33-jährige Französin

Professorin für Mathematische Statistik und Maschinelles Lernen an der Otto-von-Guericke-Universität Magdeburg. Ihr Kollege Timo de Wolff hat sie Mitte Dezember letzten Jahres online „getroffen“, um mehr über ihr Arbeitsgebiet und ihre wissenschaftlichen Interessen zu erfahren, aber auch um über Verantwortung in der KI-Forschung und Diversität in der Mathematik zu sprechen.
\end{abstract}

Herzlichen Glückwunsch zum Preis! Wir führen das Interview heute virtuell, deshalb meine erste Frage: Wie geht es Dir und Deiner Arbeitsgruppe?

Die Arbeit ist im Moment natürlich anstrengender, so wie für alle. Aber wir haben uns ein bisschen umorganisiert und treffen uns online. Das klappt gut. Weil wir in der Forschung alle sehr mobil sind, wird es, wenn wir uns erst mal an die Online-Werkzeuge gewöhnt haben, einfacher werden sich zu treffen. Das ist der positive Aspekt. Wie ist das bei Euch in der Gruppe?

Bei uns ist es ähnlich. Wir treffen uns virtuell, das ist für alle herausfordernd. Aber es ist sehr schön, dass wir jetzt mehr virtuelle Seminare haben, an denen mehr Leute teilnehmen können oder wo man, auch wenn man weit weg ist, schnell mal vortragen kann.

Ja, auch aus ökologischen Gründen ist es wahrscheinlich gut, ein paar weniger Präsenzseminare oder Konferenzen zu haben und dafür mehr online. Wir fliegen sehr viel und es ist gut, wenn wir das in der Zukunft weniger machen.

Da stimme ich Dir zu. Reden wir jetzt ein bisschen über Mathe. Erzähle uns doch bitte, worüber du forscht.

Ich arbeite mit Methoden der mathematischen Statistik und des maschinellen Lernens zu ganz verschiedene Modellen und Themen. Es gibt da nicht wirklich ein einziges Modell, für das ich mich interessiere, sondern relativ viele. Aber es gibt etwas, das alles miteinander verbindet, und das ist die Minimax-Theorie. Auf diesem Gebiet nimmt man Daten und formuliert eine statistische Fragestellung zu diesen Daten. Und die Frage, die ich gerne stelle, lautet: Existiert überhaupt eine Methode, die das bewirkt, was man erreichen möchte? Und kann man das dann auch mathematisch beweisen? Was ist das Minimum an Daten, das man braucht, um eine solche Methode anzuwenden? Das heißt, man stellt sich fundamentale Fragen darüber, was die Daten uns sagen.
Du hast gesagt, dass Du sowohl in der Statistik als auch im maschinellen Lernen zu Hause bist. Was war ursprünglich Dein fachlicher Hintergrund?

Das war maschinelles Lernen. Ich habe meinen PhD am Centre INRIA Lille Nord-Europa in Frankreich gemacht, also an der Schnittstelle von Mathematik und Informatik. Ich habe dort in einer Gruppe geforscht, die Teil der Maschinelles-Lernen-Community war, und auch meine ersten Veröffentlichungen sind ausschließlich auf diesem Gebiet. Erst nach der Promotion habe ich entschieden, eher in Richtung mathematische Statistik weiterzuarbeiten.

Und was hast Du vorher gemacht, also im Studium?

Ich habe an einer der kleineren Grandes Ecoles in Frankreich, an der ENSAE (École Nationale de la Statistique et de l'Administration Économique) studiert, wo Statistik und Wirtschaftswissenschaften gelehrt werden. Dort habe ich viel klassische Statistik gelernt, aber das hat mich damals nicht so sehr interessiert. Wahrscheinlichkeitstheorie hat mich mehr interessiert, und Informatik mochte ich auch ganz gerne. Also habe ich gedacht, okay, dann ist maschinelles Lernen eigentlich perfekt für mich. Damals gab es auch nicht so einen Hype darum wie heute. Als ich studiert habe, war Finance der große Hype. Und das hat mich überhaupt nicht gereizt. Ich habe mich für maschinelles Lernen entschieden, weil ich dachte, das ist ein kleines Gebiet, da gibt es nicht so viele Leute. Das hat sich inzwischen geändert [lacht].

$\mathrm{Zu}$ Beginn unseres Gesprächs hast Du die Fragen erwähnt, die Dich in Deiner Forschung umtreiben. Könntest Du das bitte noch einmal an einem Beispiel oder einer konkreten Anwendung erläutern. Spontan fällt mir dazu Matrix Completion ein.

Genau, ein Beispiel dafür sind Recommender Systeme. Das sind die Programme oder Algorithmen, die auf Internetseiten Empfehlungen für Benutzer generieren. Google 


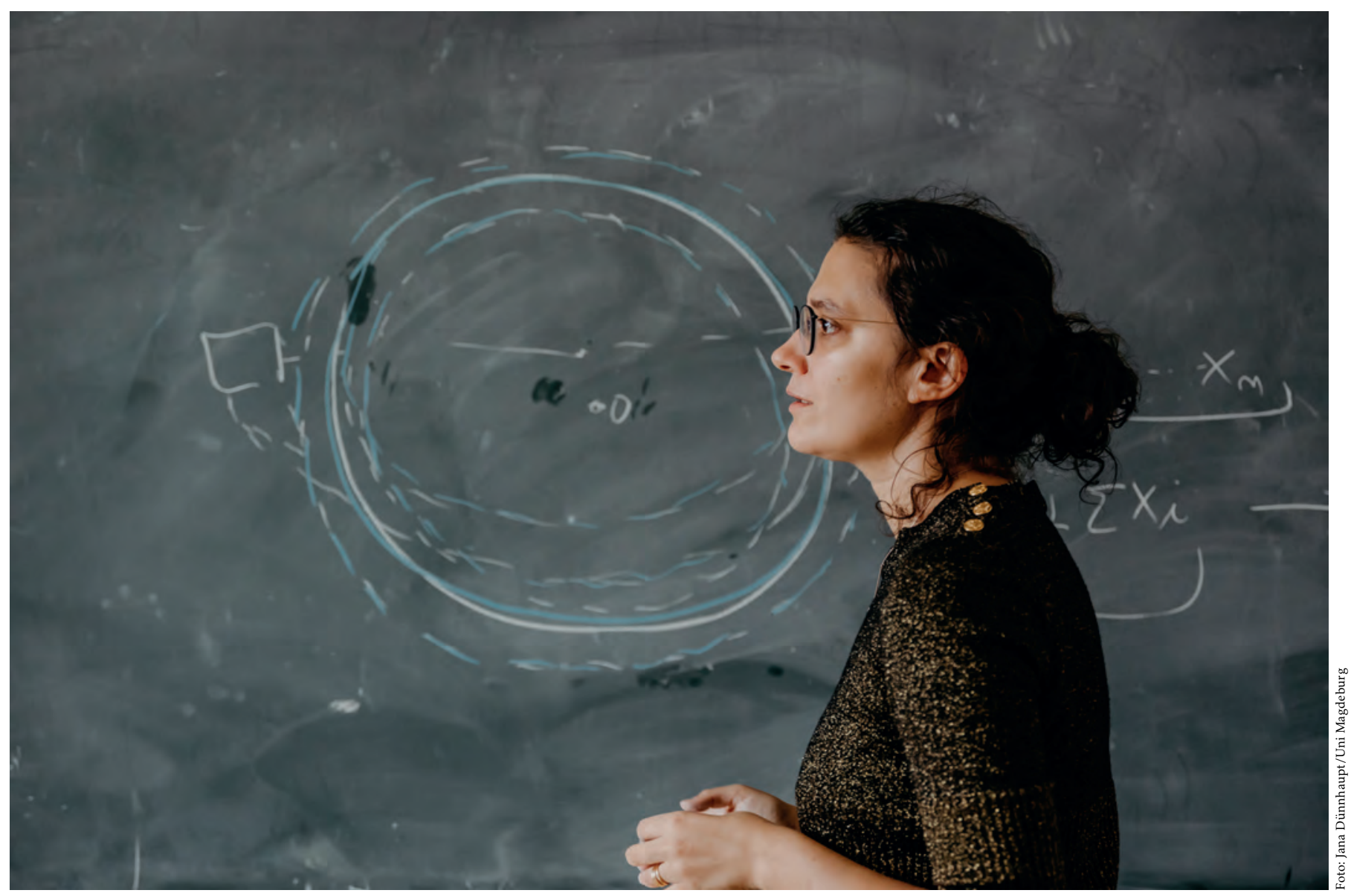

Alexandra Carpentier

Search ist so ein Recommender System. Oder wenn einem in einem Online-Shop etwas automatisch angeboten wird, dann ist das auch ein Recommender System, das entscheidet, was angeboten wird. Ich selbst programmiere nicht und konstruiere auch keine Recommender Systeme, aber ich arbeite mit Kollegen in der Industrie zusammen, die da ein bisschen näher dran sind. Was ich mache, ist die Entwicklung von Algorithmen, aber wirklich nur die Theorie. Und Matrix Completion, das stimmt, ist ein Problem, das mit Recommender Systemen verknüpft ist, denn typischerweise hat man dort eine große Datenbasis. Und diese Daten sind in einer Art Tabelle nach Produkten und nach Nutzern organisiert. Man weiß also, man hat dieses oder jenes diesem oder anderen Benutzern angeboten und sie haben das gemocht oder nicht gemocht. Jetzt hat man diese große Tabelle, in der ein paar Felder gefüllt sind, aber ganz viele sind leer, weil man bestimmte Benutzer nie registriert oder ihnen nie ein Produkt angeboten hat. Bei Matrix Completion ist nun die Frage, wie man die Tabelle mit Hilfe statistischer Methoden füllt. Man möchte abschätzen, was ein Benutzer über ein Produkt denken wird, obwohl er dieses Produkt noch nicht gesehen hat. Und das kann man machen, weil dieser Benutzer beispielsweise ein anderes Produkt gemocht hat oder weil ein anderer Benutzer es gemocht hat, denn man kann davon ausgehen, dass es Ähnlichkeiten zwischen Benutzern gibt. Das ist, grob gesagt, die Idee hinter Matrix Completion. Ein anderes Gebiet, auf dem ich eigentlich viel mehr forsche als über Matrix Completion, nennt sich Bandit-Theorie. Bei diesem Modell stellt man sich vor, dass man in ein Casino geht und dort gibt es diese Einarmigen Banditen. Man stellt sich vor, dass ein paar besser sind als andere.

\section{Was heißt hier besser?}

Von denen bekommt man mit höherer Wahrscheinlichkeit Geld - so stellt man sich das zumindest vor. Der Benutzer möchte sobald wie möglich und so viel wie möglich auf der besseren Maschine spielen. Daher probiert er ein bisschen rum und konzentriert sich dann auf die beste Maschine. Die Bandit-Theorie ist eigentlich ein Gebiet der Algorithmenentwicklung und mit deren Hilfe macht man diese Art von Optimierung. Und die ist auch für Recommender Systeme sehr wichtig. Denn man möchte sich so schnell wie möglich auf die besten Produkte konzentrieren. Darüber forsche ich und das war auch das Thema meiner Doktorarbeit. Es liegt an der Schnittstelle von 
Optimierung und Statistik und ich arbeite mit den Mitteln des maschinellen Lernens - und damit ist es auch ein Randgebiet der Informatik.

Da muss ich jetzt nachfragen, denn in der Optimierung bin ich jetzt ja selber ein bisschen zu Hause. Inwiefern sind denn in so einem Bandit-Szenario, wie Du es gerade beschrieben hast, die verschiedenen Optimierungsverfahren wichtig?

Die Bandit-Theorie liegt wirklich an der Schnittstelle von Statistik und Optimierung. Allerdings ist es eine Art von Optimierung, die vielleicht nicht ganz Mainstream ist. Es geht weniger um konvexe Optimierung und am meisten um diskrete Optimierung, also eher ein Randgebiet der Optimierung.

Ich glaube, es kommt darauf an, wen man fragt. Ich bin mir sicher, es gibt viele Leserinnen und Leser, für die das sehr zentral ist.

Ja, natürlich, es gibt viele Optimierer, die das machen. Aber wenn man Optimierung unterrichtet, dann ist das nicht das erste Thema, das drankommt. Bandit-Theorie liegt eben etwas mehr am Rand, weil es nicht an sich Kombinatorik ist. Es geht mehr in Richtung ExplorationExploitation Trade-Off, wegen dem Rauschen in den Daten. Deshalb liegt Bandit-Theorie ein bisschen näher an der Statistik, also am maschinellen Lernen als in der Optimierung.

Gibt es auf dem Gebiet der Bandit-Theorie ein bestimmtes Resultat, auf das du hinarbeitest, ein bestimmtes Theorem, das du gerne beweisen würdest?

Was ich im Moment ganz, ganz spannend finde in der Statistik sind Fragen, wo man ein Modell in hoher Dimension hat, also ein sehr komplexes Modell. Und dann stellt man sich eine Frage über dieses Modell und diese komplexen Daten, die weniger kompliziert ist. Man fragt sich, was man am besten machen kann, um diese Frage zu beantworten, und wenn man das tut, gibt es zwei unterschiedliche Kräfte, die sich bekämpfen. Einerseits die Tatsache, dass der Raum sehr hochdimensional ist und somit das Problem schwieriger, und andererseits die Tatsache, dass die Fragestellung selbst eigentlich gar nicht so kompliziert ist. Man könnte also annehmen, dass es eine einfache Antwort gibt. Tatsächlich gibt es aber sehr viele einfache Probleme, die sehr schwer zu lösen sind. Ein Problem, das wir im Moment gemeinsam mit einem Kollegen und mit einem Doktoranden bearbeiten, ist folgendes: Wenn man eine Tabelle mit ein paar Fragen hat und mit ein paar Experten, die diese Fragen beantwortet haben, dann möchte man wissen, wer die besten Experten sind, um die Tabelle entsprechend zu ordnen. Dafür prüft man die Antworten, und die vertrauenswürdigen Experten haben ein paar mehr Fragen richtig beantwortet. Mich interessiert im Moment, wie man diese Tabelle am besten ordnen kann. Wenn die Anzahl von Experten zum Beispiel kleiner ist als der Anzahl der Fragen, dann könnte man sich denken, dass es einfacher ist oder schneller geht, wenn man die Experten ordnet anstatt die gesamte Matrix zu schätzen. Bei diesem Problem stellen sich zunächst statistische Fragen, aber auch viele Optimierungsfragen. Wie kann man hier eine effiziente Methode erarbeiten? Das ist eine sehr interessante Frage, finde ich.

Schon bevor Du als Professorin für Mathematische Statistik und Maschinelles Lernen nach Magdeburg gekommen bist, warst Du im Emmy Noether-Programm der DFG. In dem Rahmen haben wir uns auch kennengelernt. Habt ihr in Deiner Forschungsgruppe auch über solche Fragestellungen gearbeitet?

In der Gruppe ging es um die Frage, wie man Algorithmen entwickeln kann, die Entscheidungen in einer Umgebung treffen, in der es viele Unsicherheiten gibt. Eigentlich sollte man in dieser Umgebung die Unsicherheit ein bisschen beschränken, um Entscheidungen treffen zu können. Aber wenn die Umgebung komplex ist, dann hängt die Unsicherheit genau von dieser Komplexität der Umgebung ab. Und je komplexer die Umgebung, desto größer die Unsicherheit. Wenn man dann die Komplexität der Umgebung nicht kennt, ist es ganz schwer zu wissen, wie hoch die Unsicherheit ist. Dieses Problem hat viel mit komplexen Fragen der Statistik zu tun. Wir arbeiten noch daran, weil das Programm noch läuft, und wir sind sehr gut vorangekommen. Inzwischen gibt es mehr und mehr Gruppen, die daran arbeiten. Das ist schön. Das Emmy Noether-Programm ist wirklich super. Ich mache hier ein bisschen Werbung [lacht].

Ja, das kann ich nur unterschreiben. Kommen wir zu einer anderen Frage. Nehmen wir an, eine BachelorStudentin liest dieses Interview und sagt, das ist ja super interessant, ich möchte gerne genau auf dem Gebiet von Professorin Carpentier forschen. Wie könnte sie sich im Studium darauf vorbereiten?

Auf meinem Gebiet ist ein Verständnis der Grundlagen der Mathematik eine wichtige Voraussetzung: ein bisschen Algebra, ein bisschen Analysis und unbedingt Wahrscheinlichkeitstheorie. Klassische Statistik ist tatsächlich nicht so hilfreich, denn da geht es sehr viel um parametrische Statistik. Ansonsten sind auch maschinelles Lernen und die Methoden der modernen Statistik eine gute Vorbereitung. Man muss aber nicht an sehr spezialisierten Vorlesungen teilgenommen haben. Für mich ist am wichtigsten, dass jemand die Bereitschaft hat, wirklich über Fragen nachzudenken und nicht nur etwas auswendig zu lernen. Wenn man ein Paper liest, sollte man sich fragen: Wie hätte ich selbst auf die Lösung kommen können? Man versucht zu verstehen, auf welche Gedanken man eben nicht gekommen ist. Das ist für mich das Wichtigste und das ist auch das, was wir in meiner Gruppe machen. Das kostet unglaublich viel Zeit, aber es macht die Forschung auch spannend.

Heißt das, dass es einfacher ist, Zugang zu bekommen, wenn man eine klassisch mathematische Ausbildung hat als wenn man aus der Informatik kommt? Oder ist 


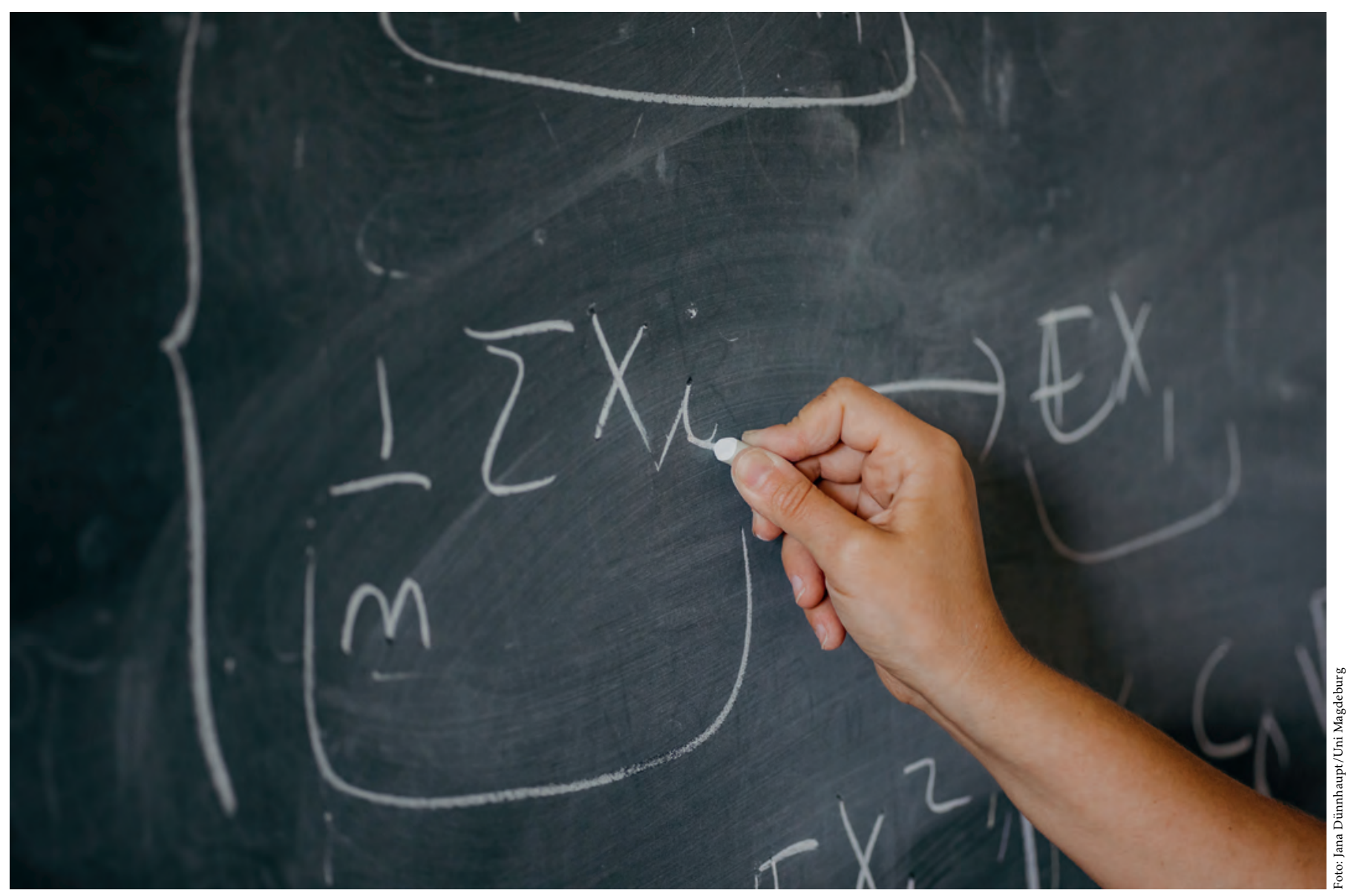

das am Ende egal, solange man das beherzigt, was Du zuletzt gesagt hast?

Ich denke, wenn man mit genügend mathematischem Background in der theoretischen Computer Science gearbeitet hat, dann ist das eine gute Voraussetzung. Wenn man aus der Mathematik kommt, dann kommt es darauf an, dass man sich erst mal mit den einfachen Problemen beschäftigt. Ich habe den Eindruck, dass Leute in der Computer Science manchmal nicht genügend mathematische Werkzeuge zur Verfügung haben, während die Studierenden in der Mathematik häufig die Haltung haben, oh, es gibt hier diese sehr komplexe Theorie, die möchte ich unbedingt anwenden, auch wenn das gar nicht nötig wäre. Und diese Art zu denken sollte man - zumindest auf meinem Gebiet - vermeiden und wirklich versuchen, sich auf die einfachen Fragen zu konzentrieren.

Nach der reinen Mathematik würde ich gerne über den breiteren, gesellschaftlichen Kontext von künstlicher Intelligenz sprechen. Das Thema KI ist ja nicht nur medial omnipräsent, es bewegt die Menschen auf der ganzen Welt im Hinblick auf die Chancen und im Hinblick auf Risiken. Wie blickst Du in die Zukunft? Siehst Du eher Chancen oder eher Risiken? Und worauf müssten wir als Gesellschaft besonders achten?
Das ist eine ganz schwierige Frage. Da habe ich nichts besonders Kluges zu sagen. Es ist unsicher, was in der Zukunft passieren wird. Aber diese Entwicklung, dass es mehr und mehr Algorithmen gibt, dass mehr und mehr Prozesse automatisiert werden, die werden wir nicht $\mathrm{zu}$ rückdrehen können. Das wird bleiben. Ich weiß auch gar nicht, ob wir etwas dagegen tun sollten, denn eigentlich ist es auch schön, wenn Maschinen für uns arbeiten. Aber es stellen sich viele andere Fragen: Zum Beispiel wie Unternehmen, die diese Automatisierung nutzen, kontrolliert werden können? Wie sollte man die Gewinne, die sie damit erwirtschaften, in der Gesellschaft verteilen? Wie könnten Gesetze aussehen? Das sind sehr komplexe Fragen - ich bin froh, keine Politikerin zu sein. Als Theoretikerin stellen sich mir diese Frage nicht direkt, aber ich habe Kontakt zu Kollegen in der Industrie und die Entwicklung dort gibt mir natürlich auch zu denken. Daher würde ich im Moment auch nicht gerne in die Industrie gehen, denn ich weiß nicht, wo das Ganze hingeht. Aber ich denke, die Frage lautet nicht weitergehen oder nicht, sondern wie gehen wir weiter und was müssen wir dafür tun? Eine Antwort darauf habe ich nicht.

Okay, aber gibt es hier nicht auch eine Verantwortung der Wissenschaft? 
Ja, sicherlich. Aber darauf sind wir nicht gut vorbereitet. Denn, wenn man in der Theorie forscht, ist man weit weg von den Anwendungen. Man hat keine sehr genaue Vorstellung davon, wie Dinge konkret implementiert werden. Das Problem auf meinem Gebiet ist nicht der Algorithmus selbst, das Kernproblem ist die Datenverwaltung. Und da kenne ich mich nicht gut genug aus. Man müsste Rechtsexperte sein und darüber hinaus auch mit der Ingenieursperspektive derjenigen vertraut sein, die die Algorithmen entwickeln. Aber es ist schwer, diese verschiedenen Leute zusammenzubringen, auch weil sie ganz verschiedene Fachsprachen sprechen. Ich denke, viele Leute möchten in dieser Richtung etwas unternehmen, aber die meisten fühlen sich ziemlich ohnmächtig. So wie ich auch. Ich würde gerne etwas beitragen, aber ich wüsste nicht, wo man anfangen könnte.

Du hast gesagt, dass Du mit Kollegen in der Industrie zusammenarbeitest. Ich habe den Eindruck, dass sich zumindest in Deutschland - die Academia in der Mathematik dem maschinellen Lernen zuerst und vor allem von der theoretischen Seite her angenähert hat und die Industrie sehr stark von der Anwendungsseite her. Trügt der Eindruck?

Die Antwort hängt ein bisschen davon ab, was man unter maschinellem Lernen versteht. Ich habe den Eindruck, dass es durch den KI-Hype in Deutschland viele Kollegen gibt, die zwar Fragen mit Mitteln des maschinellen Lernens beantworten, sich aber nicht als ML-Forscher verstehen und auch nicht in den entsprechenden Journalen oder Konferenzen veröffentlichen. Und wenn man dann guckt, welche Leute in den wichtigsten ML-Fachzeitschriften veröffentlichen, dann kommen die eher aus der Informatik. Die arbeiten dann aber weniger theoretisch als beispielsweise in Frankreich.

Ja, das würde ich auch so sehen. Und wie ist es Deiner Erfahrung nach in der Industrie?

In der Industrie - zumindest in den Unternehmen, die ich kenne, also die großen Tech Unternehmen wie Google, Amazon oder Facebook - gibt es mehr und mehr Prestigeprojekte. Das heißt, die stellen mehr und mehr Leute ein, die Theoretiker sind. Die sind nicht unbedingt an der Algorithmenentwicklung beteiligt, die machen, was sie wollen, und ihre Haupttätigkeit ist zu veröffentlichen. Ich würde sagen, Wissenschaftler in der Industrie arbeiten nicht unbedingt angewandter als die in der Academia. Was ich beobachte, ist, dass es in Deutschland weniger Verbindungen in die Industrie gibt als in Frankreich. Auf meinem Forschungsgebiet wechseln hier weniger Kollegen zu den großen Tech Firmen als in Frankreich. In meinem früheren Lab am Centre Inria Nord Europe in Lille, wo ich promoviert habe, sind inzwischen fast alle in die Industrie gegangen. Und das hat sicher viel damit zu tun, dass Wissenschaftler in Deutschland, wenn sie Tenure [einen unbefristeten Arbeitsvertrag] haben, mehr Vorteile genießen als in Frankreich. Andererseits interessieren sich diese Unternehmen eben für Leute, die in den einschlägigen Publikationen für maschinelles Lernen veröffentlichen. Und so viele Mathematiker machen das in Deutschland nicht. Das sind eher Informatiker, und die bekommen dann auch viele Angebote.

Zum Schluss würde ich gerne über das Thema Diversität sprechen. Mathematik ist in Deutschland ein Fach, das tendenziell von älteren weißen Männern dominiert wird. Du bist - auch als jemand, die nicht in Deutschland aufgewachsen ist - dagegen ein leuchtendes Beispiel dafür, wie divers Exzellenz ist. Wie beurteilst Du die Lage in Deutschland. Was ist gut und vor allem, was müsste besser werden?

Ich weiß nicht, ob ich ein Beispiel für Exzellenz in dieser Hinsicht bin, aber ich habe definitiv eine Perspektive von außen, weil ich nicht in Deutschland aufgewachsen bin. Und als Frau in der Mathematik ist man vielleicht nicht unbedingt einsam, aber es stimmt, es gibt nicht so viele Mathematikerinnen. Was kann man dazu sagen? Ehrlich gesagt, ich finde es als Frau manchmal nicht ganz einfach. Ich bin Französin, also bin ich daran gewöhnt, von Franzosen diskriminiert zu werden und nicht von Deutschen [lacht]. Die Wege in Deutschland zu diskriminieren sind anders als in Frankreich. Ich war wirklich schockiert, als ich nach Deutschland gekommen bin, das muss ich zugeben. Denn die Hierarchien sind hier viel sichtbarer, viel direkter, und als Frau kriegt man das eben eher mit denn als Mann, weil man als Frau meistens ein bisschen weiter unten in der Rangordnung zugeordnet wird. Was mir in Deutschland gefällt, ist, dass es von den Unis und auch von der DFG und der Politik den Versuch gibt, die Verantwortlichen zu zwingen, mehr Frauen in die Forschung zu bringen. Und das ist gut, denn von alleine wird das nicht klappen. Die Vorurteile gegenüber Frauen sind unglaublich stark. Das habe ich selbst oft erlebt. Als Frau wird man von bestimmten Leuten - nicht von allen, aber auch von manchen, die eine sehr hohe Stellung haben herabgesetzt und das hat natürlich Auswirkungen darauf, wie man wahrgenommen wird. Deswegen ist es gut, dass es Druck von der Politik gibt, um das ein bisschen zu kompensieren. Zum Beispiel finde ich diese Aktion von der DFG sehr effektiv, zu sagen: Gut, wenn Sie ein Projekt beantragen möchten, dann müssen unter den Principal Investigators auch Frauen sein, sonst wird der Antrag nicht durchgehen. Solche Maßnahmen gehen in die richtige Richtung. Und wenn es erst eine kritische Masse von Leuten mit einem diverseren Hintergrund gibt, dann wird sich das von alleine weiterentwickeln.

In Deutschland verlassen aber viele sehr begabte jüngere Forscherinnen schon zu einem deutlich früheren Zeitpunkt ihrer Karriere das akademische System, etwa nach dem Master oder nach der Promotion. Was kann man tun, um diesen Exodus zu verhindern.

Ja, das Problem ist ziemlich spezifisch für Deutschland, weil es, zumindest was die Mentalität angeht, aber auch die Organisation, immer noch schwer ist, Kinder zu kriegen und einen Beruf zu haben, der sehr viel Zeit in Anspruch nimmt. Das ändert sich gerade, weil es mehr und 
mehr Kitas gibt. Und es ist mehr und mehr akzeptiert in der Gesellschaft, Kinder in der Kita zu haben, ohne Rabenmutter genannt zu werden. Ein anderer Aspekt ist, dass es nicht angenehm ist, wenn man in der Community diskriminierenden Kommentaren ausgesetzt ist oder andere Probleme bekommt, weil man eine Frau ist. Deshalb gibt es sicher auch viele Frauen, die sich denken, das lohnt sich nicht, dann gehe ich halt weg. Aber wenn diese Diskriminierung weniger wird und auch das Problem der Kinderbetreuung einfacher zu lösen ist, dann werden auch mehr Frauen in dem Beruf bleiben.

Eine letzte Frage dazu: Du hast selbst Diskriminierung erfahren als Frau, aber Du bist geblieben. Gibt es etwas, was Du den jüngeren Forscherinnen mitgeben kannst, damit sie sich nicht entmutigen lassen?

Das ist eine schwierige Frage. Wenn jemand einen blöden Kommentar macht, dann sollte man sich als Erstes sagen, dass das nicht gegen mich als Person gerichtet ist, sondern gegen mich als Frau oder als Minorität. Und dann sollte man am besten versuchen darüber zu lachen. Mir passiert es zum Beispiel immer wieder, dass Leute bei Konferenzen denken, dass ich zum Servicepersonal gehöre. Das ist der Klassiker. Oder es gab Teilnehmer, die mir ausführlich erklärt haben, dass ich da eigentlich nichts zu suchen habe, dass es Zeitverschwendung wäre für mich und für die mathematische Community, weil es eigentlich Wichtigeres für mich zu tun gäbe, zum Beispiel sich um Babys zu kümmern oder in der Küche zu arbeiten. Wie kann man darüber lachen? Indem man sich bewusst macht, wie lächerlich das Ganze ist. Man kann sich auch irgendwelche passenden Namen für solche Leute ausdenken. Ich kenne keine Zaubertricks, aber ich denke, darüber zu lachen ist besser, als sich nur darüber zu ärgern. Es ist nur eine Minderheit von Leuten, die es einem unangenehm macht. Aber, ja, es passiert immer wieder, insbesondere jungen Kolleginnen. Ich würde ihnen raten, aufmerksam zu sein, wenn sie so etwas beobachten. Sie sollten dann entweder direkt etwas sagen oder danach zu der Kollegin gehen und sich erkundigen, wie es ihr geht. Denn solche Situationen sind sehr anstrengend und wenn man ein bisschen Unterstützung bekommt, fühlt man sich gleich besser. Es ist wichtig, die Gewissheit zu bekommen, dass das, was man gerade erlebt hat, nicht angemessen war. Danach kann man auch besser darüber lachen. Und wenn man jemanden hat, mit dem man lachen kann, umso besser.

Kommen wir noch einmal zum Preis zurück, den Du gewonnen hast. Der von Kaven-Ehrenpreis ist mit 10 ooo Euro dotiert. Was hast Du vor mit dem Geld?

Ich weiß noch nicht, was ich damit machen werde. Vielleicht werde ich meine Doktoranden und Postdocs zu Gruppentreffen hier in Magdeburg einladen, damit sie sich noch besser kennenlernen und untereinander vernetzen können. Solche Dinge sind nicht abgedeckt durch das Budget des Lehrstuhls.

Prof. Dr. Timo de Wolff,

Institut für Analysis und Algebra,

Technische Universität Braunschweig,

Universitätsplatz 2, 38106 Braunschweig

t.de-wolff@tu-braunschweig.de

Timo de Wolff ist seit 2019 W2-Professor für Angewandte Algebra an der TU Braunschweig. Vorher war er an der Goethe Universität Frankfurt am Main, der Universität des Saarlandes, der Texas AEM Universität und der TU Berlin tätig. Seit 2017 ist er Mitglied im Emmy Noether Programm der DFG. Seit 2019 ist er gewähltes Mitglied der Jungen Akademie an der BBAW und der Leopoldina. 